\title{
Comparing Prescription Sales, Google Trends and CDC Data as Flu Activity Indicators
}

\author{
Avinash Patwardhan* and David Lorber \\ Clinical Affairs, Walgreen Co., Deerfield, IL, USA
}

\section{Objective}

To examine if the prescription sales data from a large retail pharmacy chain in the US were comparable to Google Flu trends and CDC's US ILI Network data as flu activity indicator.

\section{Introduction}

In a 2007 survey of public health officials in the United States, International Society for Disease Surveillance found that only $7 \%$ used pharmacy prescription sales data for surveillance (1). There have been many reports suggesting effective use of prescription sales data in syndromic surveillance $(2,3,4,5)$. Community pharmacies can provide a valuable supplementary tool for syndromic surveillance of infectious diseases.

\section{Methods}

We extracted five years of de-identified prescription sales data from the proprietary pharmacy computer system of a large retail pharmacy chain in the United States. The prescriptions were written for the common drugs for the treatment of influenza: Amantadine, Oseltamivir, Rimantadine, and Zanamivir. We acquired Google Flu trends national aggregate counts data that represented the estimates of the ILI cases per 100,000 physician visits. We acquired CDC ILINET data for 2007. We calculated Pearson ' $r$ ' between our data and Google and CDC data. We also created comparable trends graphs after converting the counts of the influenza scripts and the counts of the Google estimated ILI cases to logarithmic scale.

\section{Results}

The Pearson ' $r$ ' between the aggregate counts of scripts for all the four drugs and the Google estimates of the ILI cases for years 2007, 2008, 2009, 2010, and 2011 were 0.85 (95\% CI, 0.75-0.91), 0.92 (95\% CI, 0.86-0.95), 0.91(95\% CI, 0.85-0.95), 0.88 (95\% CI, $0.80-$ 0.93 ), and 0.87 (95\% CI, 0.78-0.92) and 0.92 (95\% CI, 0.90-0.94) for years 2007 through 2011 together. The Pearson ' $r$ ' between the aggregate counts of scripts and the CDC \% unweighted ILI (2007) was 0.97 (95\% CI, 0.95-0.98).

\section{Conclusions}

A strong to very strong correlation between prescription sales data and Google Flu trends and CDC's ILI activities data for influenza suggests that the former can serve as a good and valid influenza activity indicator. Community pharmacies with large footprints might want to proactively build automated syndromic surveillance systems with near real time reporting capabilities and explore the possibility of building predictive models (3) and of forming partnerships with public health agencies for syndromic surveillance.

Pearson ' $r$ ' between prescription sales data and Google Flu trends and CDC ILI data

\begin{tabular}{|c|c|c|c|}
\hline Prescription sales data and & Year & Pearson ' $\mathrm{r}$ ' & 95\% CI $(\mathrm{p}=0.05)$ \\
\hline Google Trends ILI data & 2007 & 0.85 & $0.75-0.91$ \\
\hline & 2008 & 0.92 & $0.86-0.95$ \\
\hline & 2009 & 0.91 & $0.85-0.95$ \\
\hline & 2010 & 0.88 & $0.80-0.93$ \\
\hline & 2011 & 0.87 & $0.78-0.92$ \\
\hline CDC \% unweighted ILI data & Aggregate (2007-2011) & 0.92 & $0.90-0.94$ \\
\hline
\end{tabular}

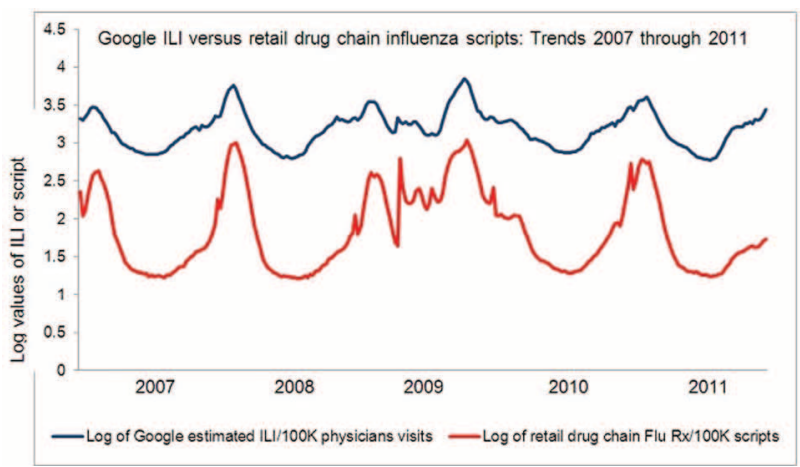

Keywords

public health; syndromic surveillance; Flu; prescription sales; activity or early warning indicator

\section{Acknowledgments}

We wish to acknowledge Criselda Valencia (Lead Analyst Support \& Deployment Data Warehouse at Walgreen Co.), Erma Villarreal (IT Manager - Support \& Deployment Data Warehouse at Walgreen Co.), and Zhongwen Huang (Senior Analyst Outcomes \& Analytics at Walgreen Co.) for pulling data for us.

\section{References}

1. The International Society for Disease Surveillance (ISDS) Available: http://www.syndromic.org/ Accessed May 2, 2012. 2. Chadwick D (2009) The Rhode Island Department of Health. The First Statewide System for Tracking Disease Using Prescription Data. Press Release Archives. State of Rhode Island. Department of Health. Available: http://www.health.ri.gov/news/archives/2009/ Published October 27, 2009. Accessed May 2, 2012. 3. Sugawara T, Ohkusa Y, Ibuka Y, Kawanohara H, Taniguchi K, Okabe N (2012) Real-time Prescription Surveillance and its Application to Monitoring Seasonal Influenza Activity in Japan J Med Internet Res. 14(1):e14. 4. Vergu E, Grais RF, Sarter H, et al. (2006) Medication sales and syndromic surveillance, France. Emerging infectious diseases. 12(3):416. 5. van den Wijngaard C, van Pelt W, Nagelkerke N, Kretzschmar M, Koopmans M (2011) Evaluation of syndromic surveillance in the Netherlands: its added value and recommendations for implementation. Euro Surveill. 16(9):19806.

\footnotetext{
*Avinash Patwardhan

E-mail: avinash.patwardhan@walgreens.com
} 